\title{
Development of a New Type of Recombinant Hyaluronidase Using a Hexahistidine; Possibilities and Challenges in Commercialization
}

\author{
Chaeri Park $^{1 \dagger}$, Yun-Kyoung Song ${ }^{1 \dagger}$, Young-Hyun Kim ${ }^{2,3+}$, Yena Jung1, Young-Ho Park ${ }^{2}$, Bong-Seok Song ${ }^{2,3}$, \\ Taekil Eom ${ }^{4}$, Ju-Sung Kim ${ }^{4}$, Sang-Hyun Kim ${ }^{5}$, Ji-Su Kim ${ }^{2,3}$, Sun-Uk Kim ${ }^{2,3}$, Sang-Rae Lee ${ }^{2,3 *}$, and Ekyune Kim ${ }^{1 *}$ \\ ${ }^{1}$ College of Pharmacy, Catholic University of Daegu, Gyeongsan 38430, Republic of Korea \\ ${ }^{2}$ National Primate Research Center and Futuristic Animal Resource and Research Center, Korea Research Institute of Bioscience and \\ Biotechnology, Republic of Korea \\ ${ }^{3}$ Department of Functional Genomics, KRIBB School of Bioscience, Korea University of Science and Technology (UST), Daejeon 34113, \\ Republic of Korea \\ ${ }^{4}$ College of Applied Life Sciences, the Research Institute for Subtropical Agriculture and Biotechnology, Jeju National University, Jeju 63249, \\ Republic of Korea \\ ${ }^{5}$ Institute of Animal Medicine, College of Veterinary Medicine, Gyeongsang National University, Jinju 52828, Republic of Korea
}

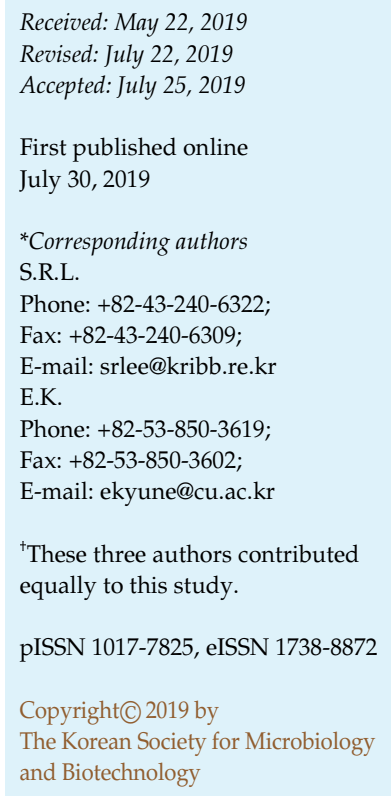

Hyaluronidases enhance therapeutic drug transport by breaking down the hyaluronan barrier to lymphatic and capillary vessels, facilitating their tissue absorption. Commercially available hyaluronidases are bovine in origin; however, they pose risks such as bovine spongiform encephalopathy. The present study aimed to develop a novel, highly active hyaluronidase and assess its function. Therefore, in order to find the most efficient active hyaluronidase, we produced several shortened hyaluronidases with partial removal of the $\mathrm{N}$ - or C-terminal regions. Moreover, we created an enzyme that connected six histidines onto the end of the hyaluronidase C-terminus. This simplified subsequent purification using $\mathrm{Ni}^{2+}$ affinity chromatography, making it feasible to industrialize this highly active recombinant hyaluronidase which exhibited catalytic activity equal to that of the commercial enzyme. Therefore, this simple and effective isolation method could increase the availability of recombinant hyaluronidase for research and clinical purposes.

Keywords: Recombinant, hyaluronidase, affinity chromatography, enzyme, hyaluronic acid
Glycosaminoglycan hyaluronic acid (HA), a polymer consisting of repeating disaccharide units of N-acetyl-Dglucosamine and D-glucuronic acid, is a major structural component of connective, epithelial, and neural tissues, and the cumulus-oocyte complex [1-3]. The mechanisms of HA synthesis and degradation are critical to multiple biological processes, including cell migration, wound healing, malignant transformation, tissue turnover, fertilization, and egg development [4-8]. Hyaluronidases, which possess endo-beta- $\mathrm{N}$-acetyl hexosaminidase activity, are responsible for the degradation of $\mathrm{HA}$, and are widely distributed from bacteria to mammals. Of six human hyaluronidases, three (HYAL1, 2, and 3) are expressed in somatic cells, whereas the other three (HYAL4 and 6, and sperm adhesion molecule 1 (SPAM1)) are expressed in germ cells and human chromosomes 3p21.3 and 7q31.3 [912]. The well-known, sperm-specific glycosylphosphatidylinositol-anchored hyaluronidase, SPAM1, has been long believed to assist sperm penetration through the cumulus mass surrounding the eggs [13,14].

Hyaluronidase is commercially available as an injectable enzyme solution that speeds the natural breakdown of HA. 
Table 1. Primer sequences for hyaluronidase fragments.

\begin{tabular}{lll}
\hline & \multicolumn{1}{c}{ Sense primer $\left(5^{\prime}\right.$ to $\left.3^{\prime}\right)$} & \multicolumn{1}{c}{ Anti-sense primer $\left(5^{\prime}\right.$ to $\left.3^{\prime}\right)$} \\
\hline Short 1 & GCCACCATGGACAGCGTGGGCTTGGCGGTC & TTAATTTTAATAGGTTGTTTGAT \\
Short 2 & GCCACCATGAGAATGCTGAGGCGCCACCA & TCACTTCACAGGGCTGTCTATAC \\
Short 3 & GCCACCATGAGAATGCTGAGGCGCCACCA & TCACCAGTGTTTCCTTGTACACA \\
His tagged & GCCACCATGAGAATGCTGAGGCGCCACCA & ATGATGATGATGATGATGTACCTTCACAGGGCTGTCTATAC \\
\hline
\end{tabular}

It can be administered subcutaneously with other medicines to improve their uptake by the body. Hyaluronidase injection is not only used to increase the absorption of fluids or medicines that are injected subcutaneously, but also to improve absorption of radioactive substances during a procedure called subcutaneous urography. Our previous study demonstrated that bovine SPAM1 is the most active hyaluronidase in mammals [15-17]. SPAM1 exhibits dual functions: hyaluronan-hydrolyzing and ZPbinding activities. Because SPAM1 is enzymatically active at both acidic and neutral $\mathrm{pH}$, the neutral enzyme is postulated to function in hyaluronan degradation of the cumulus cell layer during sperm penetration [10].

Currently, two main types of hyaluronidase are commercially available: one extracted directly from bovine testis and the other, a human recombinant hyaluronidase. Bovine hyaluronidase has merit for its activity, but may convey potential risk of disease via contamination with infectious agents such as bovine spongiform encephalopathy, because of its native animal origin. Alternatively, the recombinant human hyaluronidase produced by expression of the human SPAM1 gene in COS cells requires purification steps and generally shows low activity. Therefore, in this study, we aimed to develop a recombinant hyaluronidase with high activity, low risk of contamination, and at an easy-to-achieve, high level.

\section{Materials and Methods}

\section{DNA Cloning}

Six oligonucleotide primers were designed to amplify DNA fragments encoding various bovine SPAM1 regions (Table 1). PCR was performed with a PCR thermal cycler (Takara Bio Inc., Japan) for 35 cycles at $94^{\circ} \mathrm{C}$ for $60 \mathrm{sec}, 60^{\circ} \mathrm{C}$ for $60 \mathrm{sec}$, and $72^{\circ} \mathrm{C}$ for $120 \mathrm{sec}$. The products were cloned, sequenced, and subjected to $1.0 \%$ agarose gel electrophoresis. The desired PCR products were then purified using a gel extraction kit in accordance with the manufacturer's guidelines. Purified DNA fragments were cloned in DH5 $\alpha$ cells using the pMD20 vector kit (Takara Bio., Japan). Following plasmid DNA isolation, inserts were sequenced using the vector-specific sequencing primer on an ABI 3100 DNA sequencer (Applied Biosystems, USA). The DNA fragments were then introduced into a pCXN2 vector. HEK 293 cells were transfected with the plasmid constructs via the Lipofectamine method (Invitrogen, USA) and cultured in Dulbecco's modified Eagle medium/heat-inactivated fetal bovine serum in the presence of $0.5 \mathrm{mg} / \mathrm{ml} \mathrm{G418}$ at $37^{\circ} \mathrm{C}$ in $5 \% \mathrm{CO}_{2} / 95 \%$ air. For the HA degradation assay, the G418-resistant clones were dissolved in RIPA buffer after obtaining the supernatant.

\section{Production of Chimeric Hyaluronidase}

To construct a new type of recombinant hyaluronidase using human SPAM1 (hSPAM1) and bovine SPAM1 (bSPAM1) gene information, the plasmid pCXN2-bSPAM1 and -hSPAM1 gene fragments were used as a template (Fig. 3A). After performing the $1^{\text {st }}$ PCR using the above method, the two fragments were ligated with T4 DNA ligase in a total volume of $10 \mu$ l (Elpis Biotech, Korea). The $2^{\text {nd }} \mathrm{PCR}$ was subsequently performed with a mixture containing $0.1 \mathrm{ul}$ of the resulting ligation product, $0.5 \mu \mathrm{M}$ of each primer (Table 2), and Pfu polymerase in a total volume of $50 \mathrm{ul}$. After a denaturation step at $94^{\circ} \mathrm{C}$ for $3 \mathrm{~min}, 35$ cycles were carried out at $94^{\circ} \mathrm{C}$ for $1 \mathrm{~min}, 60^{\circ} \mathrm{C}$ for $30 \mathrm{sec}$, and $72^{\circ} \mathrm{C}$ for $90 \mathrm{sec}$. The target DNA fragment was obtained by gel purification of each PCR product inserted into the pCXN2 and sequenced.

\section{His-Tagged Recombinant Hyaluronidase}

To express His-tagged bSPAM1 hyaluronidase, DNA fragments encoding bSPAM1 were amplified via PCR, ligated into the pCXN2 vector connected poly-histidine and then transfected into HEK293 cells using Lipofectamine 2000. Cells were then cultured for two days in Dulbecco's modified Eagle's medium containing $10 \%$ fetal bovine serum. HEK293 cells expressing the His-tagged proteins were lysed in phosphate buffered saline (PBS) containing $1 \%$ Triton X-100 and $1 \%$ protein inhibitor cocktail. After

Table 2. Primer sequences for chimeric hyaluronidase.

\begin{tabular}{lll}
\hline & \multicolumn{1}{c}{ Sense primer $\left(5^{\prime}\right.$ to $\left.3^{\prime}\right)$} & Anti-sense primer $\left(5^{\prime}\right.$ to $\left.3^{\prime}\right)$ \\
\hline Human & gccaccatgggagtgctaaaattcaagca & tattacaattccagaagcacccag \\
Bovine & tggggcagtctcaatctaagctta & ctagaggtgatggtgatgatgcttcaca \\
\hline
\end{tabular}


centrifugation $(16,000 \times g)$ for $10 \mathrm{~min}$, the recombinant protein was affinity-purified on a His-binding agarose resin column (Elpis Biotech, Korea), as previously described [8]. The sample was then subjected to SDS-PAGE to analyze the activity of recombinant hyaluronidase through interactions between poly-histidine and $\mathrm{Ni}^{2+}$.

\section{Zymography}

Proteins exhibiting hyaluronidase activity were visualized via SDS-PAGE in the presence of $0.01 \%$ human umbilical cord hyaluronic acid (HA) (Sigma-Aldrich, USA) under non-boiled and non-reducing conditions. After electrophoresis, the gels were washed with $50 \mathrm{mM}$ sodium acetate buffer, $\mathrm{pH} \mathrm{7,} \mathrm{containing}$ $0.15 \mathrm{M} \mathrm{NaCl}$ and $3 \%$ Triton X-100 at room temperature for $2 \mathrm{~h}$ to remove SDS. The gels were then incubated in the same buffer without Triton $\mathrm{X}-100$ at $37^{\circ} \mathrm{C}$ overnight. The HA-hydrolyzing proteins were detected as transparent bands against a blue background by staining the gels with $0.5 \%$ Alcian Blue $8 \mathrm{GX}$ and Coomassie brilliant blue R-250 [10].

\section{HA Dispersal Assay}

Fifty microliters of $1 \%$ HA was digested with recombinant hyaluronidase for $12 \mathrm{~h}$ in an incubator set at $37^{\circ} \mathrm{C}$. Additionally, to ascertain whether the tissues and recombinant hyaluronidase activity patterns vary according to $\mathrm{pH}$, the HA digestion assay was performed over a range of $\mathrm{pH}$ values (2-10). The digested hyaluronan samples were then separated via electrophoresis on $0.8 \%$ agarose gels. Finally, the gels were stained with $0.5 \%$ Alcian Blue $8 \mathrm{GX}$ to visualize the HA bands.

\section{Dispersal of Cumulus Cells In Vitro}

Superovulation was induced in female ICR mice (8 weeks old, Hyo-Chany Sci., Korea) with an intraperitoneal (IP) injection of pregnant mare's serum gonadotropin (5 units, Teikokuzoki, Japan) and human chorionic gonadotropin (5 units, Teikokuzoki) $48 \mathrm{~h}$ later. The cumulus mass containing eggs was collected from the oviductal ampulla of the superovulated mice $14 \mathrm{~h}$ after the injection of human chorionic gonadotropin, placed in a 50- $\mu$ l drop of TYH medium covered with mineral oil, treated with recombinant hyaluronidase, and then observed under an Olympus (Tokyo, Japan) IX71 microscope equipped with a DP-12 camera, as described [10].

\section{Results and Discussion}

To investigate the high activity level of hyaluronidase, we first constructed three fragments with encoding the $\mathrm{N}$ - and C-terminal regions (Short 1, 2, and 3), and inserted them into the pCXN2 mammalian expression vector, based on GenBank accession number NM-214011.1 (Fig. 1A). We focused on ways of producing and commercializing the newly reconstituted bovine hyaluronidase, by removing the C-terminal region possessing a very low homology with the human counterpart. A comparison of the homology between bovine SPAM1 and human SPAM1 hyaluronidase amino acids shows $63.55 \%$ overall identity, which reaches $66 \%$ on removing 80 amino acids from the C-terminal region of bovine hyaluronidase [15]. We have successfully produced three types of fragments. Interestingly, the C-terminus-deleted fragment (Short 2; recombinant (r)bSPAM1 ${ }^{\mathrm{C} 80-\mathrm{del}}$ ) was active, whereas the N-terminus-deleted fragment (Short 1; rbSPAM1 ${ }^{\text {N135-del }}$ ), and rbSPAM1 $^{\text {C158-del }}$ (Short 3), were inactive (Fig. 1B).

To examine the potential utility of $\mathrm{rbSPAM} 1^{\mathrm{C} 80-\mathrm{del}}$
A

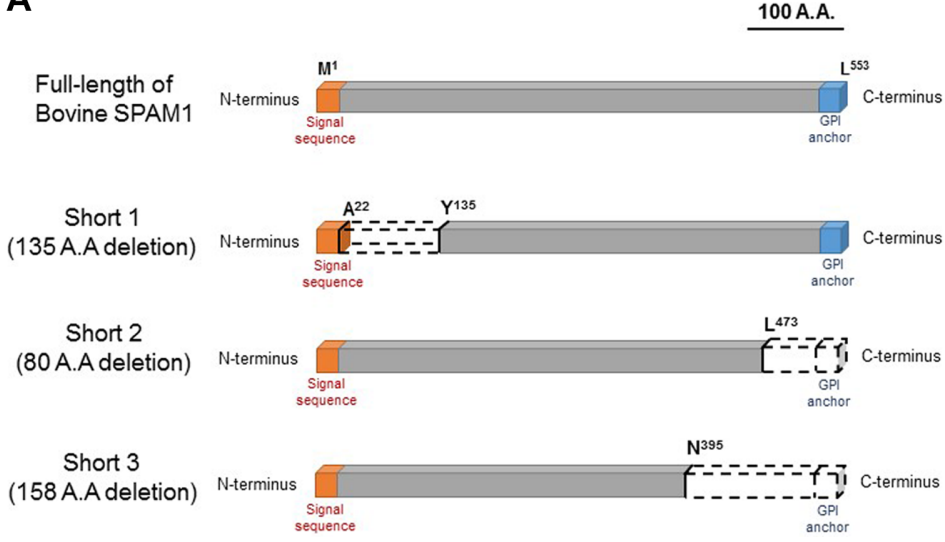

B

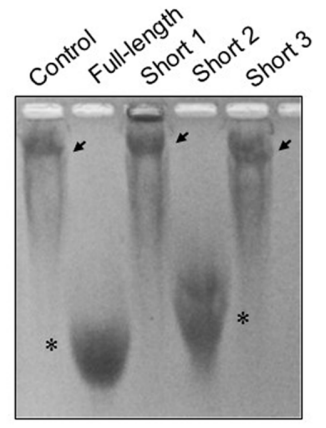

Fig. 1. Characterization of bovine sperm adhesion molecule 1 (SPAM1) hyaluronidase activity.

(A) Schematic diagrams of several bovine SPAM1 fragments. (B) Four types of fragments were transfected into HEK293 cells. After culturing, the four transfected proteins were incubated with $1 \%$ high polymer hyaluronic acid (HA) in phosphate-buffered saline. Samples were separated by $0.8 \%$ agarose gel electrophoresis. M; methionine, F; phenylalanine, $\mathrm{W}$; tryptophan, L; leucine, $\mathrm{H}$; histidine. The asterisk and arrow indicate degraded and undigested high polymer HA, respectively. 
A

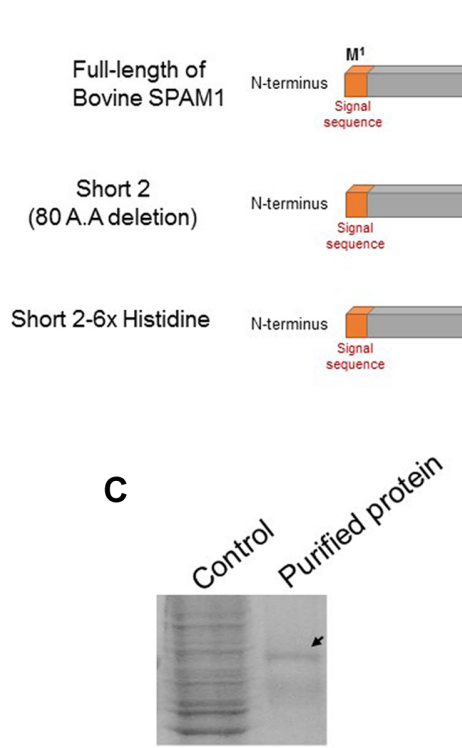

100 A.A.

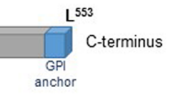

$L^{473}$

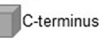

$L^{473}$ His6-tag C-terminus

\section{D}

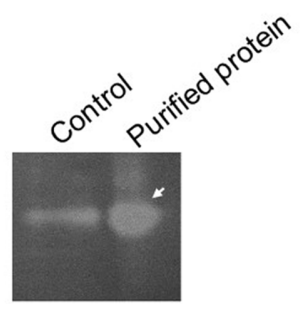

B

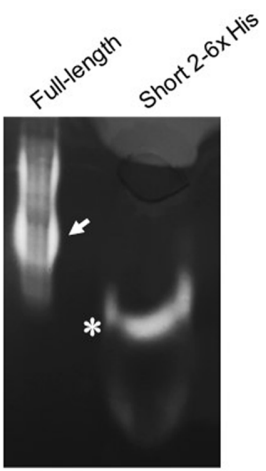

E

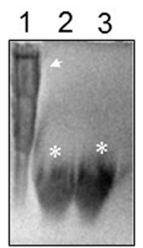

Fig. 2. Characterization of the His-tagged C-terminal-deleted recombinant bovine SPAM1 fragment (rbSPAM1 ${ }^{\text {C80-del }}$-6xH).

(A) Structural representation of rbSPAM1 ${ }^{\text {C80-del }}$ showing the poly histidine-tag fused to the C-terminus. (B) Expression of rbSPAM1 ${ }^{\text {C80-del }}-6 \times \mathrm{xH}$. rbSPAM1 ${ }^{\text {C80-del }}-6 \times \mathrm{xH}$ was detected with sodium dodecyl sulfate polyacrylamide gel electrophoresis (SDS-PAGE) containing $0.1 \%$ HA by staining with Alcian blue 8 GX. (C) The rbSPAM1 ${ }^{\text {cdel }}$-6xH hyaluronidase was purified using nickel-nitrilotriacetic acid chromatography. The purified protein was analyzed by $10 \%$ SDS-PAGE under non-reducing conditions by staining with Coomassie blue. The purified protein is indicated by the arrowhead. (D) Purified hyaluronidase separated by 1.0\% agarose gel electrophoresis followed by staining with Alcian Blue 8 GX. The white arrow head indicates digested HA. Control indicates $10 \mathrm{ug}$ of the soluble fraction from transfected HEK 293 cells. (E) Evaluation of commercial possibilities of rbSPAM1 ${ }^{\text {c80-del }}-6 \times \mathrm{xH}$ hyaluronidase. The ability to disperse HA was assessed after incubating rbSPAM1 $^{\text {C80-del }}-6 \times \mathrm{xH}$ with a high polymer of HA. 1; un-cut high polymer HA, 2; sample for rbSPAM1 ${ }^{\mathrm{C} 80 \text {-del }-6 x H}, 3$; commercial hyaluronidase.

hyaluronidase, and whether it could be produced on industrial scale, we constructed a vector with a $6 \times$ histidine- tag at the C-terminus of rbSPAM1 $1^{\text {c80-del }}\left(\right.$ rbSPAM1 $1^{\text {c80-del }}$-6xH) (Fig. 2A). Fig. 2B shows hyaluronidase activity of the
A

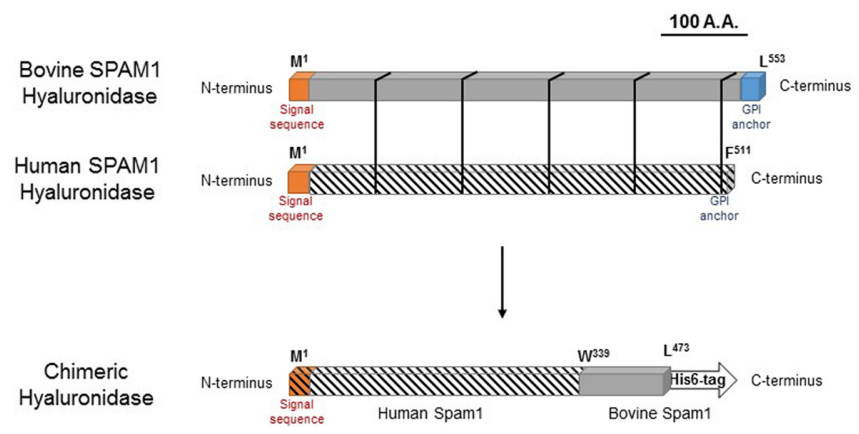

B

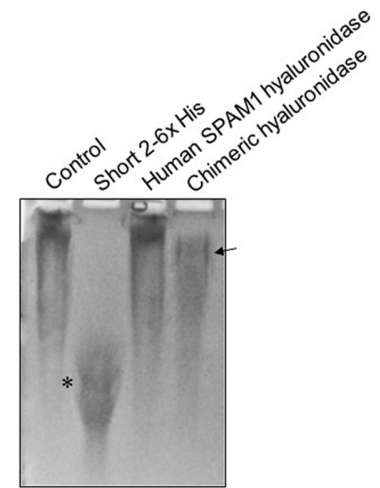

Fig. 3. Construction of chimeric hyaluronidase from human and bovine hyaluronidase.

(A) Schematic representation of bovine and human sperm adhesion molecule 1 (SPAM1). These two proteins contain four putative domains: Nterminal signal peptide, hyaluronidase catalytic, ZP-binding, and C-terminal recognition for attachment to glycosylphosphatidylinositol. Hyaluronidase chimeric cDNAs were generated by converting 339 amino acids of the N-terminus in human SPAM1 to bovine SPAM1. (B) Hyaluronidase purified by nickel-nitrilotriacetic acid chromatography was incubated with $1 \%$ high polymer HA in phosphate-buffered saline. Samples were separated by $0.8 \%$ agarose gel electrophoresis and stained with Alcian blue. The asterisk and arrow indicate HA degraded by the Short $2\left(\right.$ rbSPAM $^{\text {C80-del })}$ and chimeric hyaluronidase, respectively. 
supernatant obtained after transfection by the aforementioned method, followed by successful purification using a $\mathrm{Ni}^{2+}$ column (Figs. 2C and 2D). In addition, as shown in Fig. 2E, rbSPAM1 ${ }^{\text {C80-del }}$ hyaluronidase displayed HA dispersal ability similar to that of commercial hyaluronidase, indicating its commercial value (Fig. 2E).

Because bovine hyaluronidase has high activity but may have potential side effects, we created a chimeric hyaluronidase by combining the human and bovine proteins, utilizing human hyaluronidase in place of the $\mathrm{N}$ terminus of active hyaluronidase (Fig. 3A). Comparing the efficiency of chimeric hyaluronidase to that of rbSPAM1 $1^{\text {C80-del }}$ $6 \mathrm{xH}$ hyaluronidase (Fig. $3 \mathrm{~B}$ ), we found that its activity was lower than that of rbSPAM1 ${ }^{\text {C80-del }}-6 \mathrm{xH}$ hyaluronidase but higher than that of human hyaluronidase. We then assessed whether highly purified chimeric hyaluronidase could dissolve commercial HA-based injectable fillers used in cosmetic surgery for soft tissue augmentation, such as anti-wrinkle injections and breast enhancement. Fig. 3B

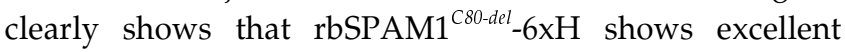
resolution of high polymer HA, whereas chimeric hyaluronidase shows low resolution. It is uncertain why the chimera showed a lower enzyme activity than the wild type; however, the structural aspect of the reconstituted hyaluronidase protein might affect the chimera activity. However, further studies need to be performed to elucidate why the chimera showed a lower enzyme activity. The rbSPAM1 ${ }^{\text {C80-del }}-6 x \mathrm{H}$ hyaluronidase exhibited an activity level equal to that of the commercial bovine native hyaluronidase in the high polymer HA dispersal assay (Fig. 2E). In addition, we confirmed that rbSPAM1 $1^{\text {C80-del }}$ $6 \mathrm{xH}$ can disperse COC (Fig. 4A). We next checked whether the rbSPAM $1^{\text {C80-del }}-6 \times \mathrm{XH}$ produced in this study was stable at various $\mathrm{pH}$ conditions ( $\mathrm{pH} 2 \sim \mathrm{pH} 10)$. The results showed no activity at $\mathrm{pH} 2$; however, the enzyme was active at other $\mathrm{pH}$ values (Fig. 4B).

Side effects of dermal HA filler injections may involve edema or tissue necrosis due to blockage of lymphatic subcutaneous small capillaries. Rapid dissolution of HA fillers by local hyaluronidase injection could mitigate such responses. To confirm the stability of rbSPAM1 $1^{\text {C80-del }}-6 \times \mathrm{xH}$, its ability to hydrolyze HA was verified under various temperature conditions. Polymeric HA decomposition was observed in samples kept at room temperature for seven days (Fig. 4C), as well as in samples that were boiled at $100^{\circ} \mathrm{C}$ (Fig. 4D). To further explore the characterization of hyaluronidase, hyaluronidase was analyzed by a hyaluronidase inhibition assay by adding 2-mercaptoethanol
A

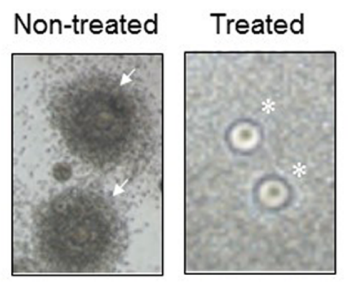

C

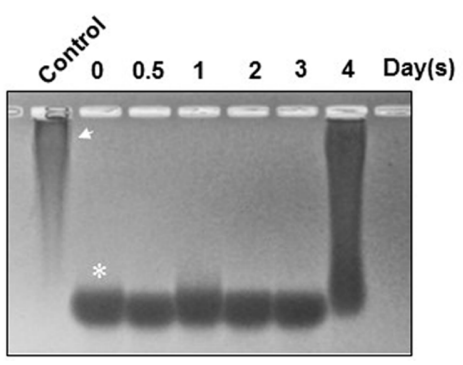

D

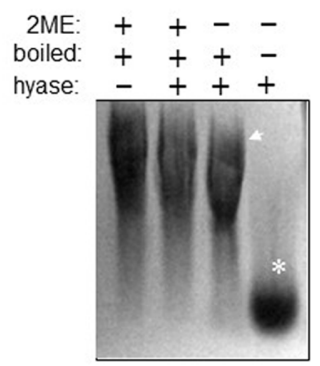

Fig. 4. Temperature stability and $\mathrm{pH}$ activity testing of recombinant his-tagged hyaluronidase.

(A) Cumulus oocyte complex (COC). COCs were incubated for 60 min with $\mathrm{rbSPAM}^{\mathrm{C} 80-\mathrm{del}}-6 \mathrm{xH}$ hyaluronidase. Asterisks indicate undispersed $\mathrm{COC}$, whereas the arrowheads indicate oocyte after COC dispersal. (B) An HA digestion assay showed activity from $\mathrm{pH} 2$ to $\mathrm{pH}$ 10. (C) rbSPAM1 ${ }^{\text {C80-del }}-6 \times \mathrm{xH}$ hyaluronidase stability evaluation at $37^{\circ} \mathrm{C}$ at various time intervals and (D) HA degradation reaction with rbSPAM1 ${ }^{\text {C80-del }}-6 \mathrm{xH}$ hyaluronidase under $1 \mathrm{mM}$ of 2-mercaptoethanol (2ME) or by boiling. The arrowhead indicates less decomposed HA. An asterisk indicates cleaved HA fragments.

(2ME). When compared with positive control, there was no hyaluronidase activity in the sample with added 2ME, despite the slight hyaluronidase activity in the boiled samples (Fig. 4D).

Thus, our data obtained from the chimeric hyaluronidase construction suggest that the disulfide bonds involved in formation of the protein structure are important for the activity of the recombinant hyaluronidase enzyme. This study was performed to determine whether the newly developed recombinant hyaluronidase showed stable activity at room temperature; if so, the enzyme would have high commercial value. In conclusion, our results suggest that $\mathrm{rbSPAM} 1^{\text {c80-del }}-6 \mathrm{xH}$ has the potential to rapidly dissolve a high polymer HA-based substance, indicating its effective application in cosmetic surgery, in vitro fertilization, and drug delivery. 


\section{Acknowledgments}

This research was supported by the National Research Foundation of Korea Grant, which is funded by the Korean Government (NRF-2017R1D1A1B03031420) and KRIBB Research Initiative Program (KGM4251913), Republic of Korea.

\section{Conflict of Interest}

The authors have no financial conflicts of interest to declare.

\section{References}

1. Zhu Y, Kruglikov IL, Akgul Y, Scherer PE. 2019. Hyaluronan in adipogenesis, adipose tissue physiology and systemic metabolism. Matrix Biol. 78-79: 284-291.

2. Camaioni A, Salustri A, Yanagishita M, Hascall VC. 1996. Proteoglycans and proteins in the extracellular matrix of mouse cumulus cell-oocyte complexes. Arch. Biochem. Biophys. 325: 190-198.

3. Viola M, Karousou E, D'Angelo ML, Caon I, De Luca G, Passi A, et al. 2015. Regulated Hyaluronan Synthesis by Vascular Cells. Int. J. Cell Biol. 2015: 208303.

4. Aya KL, Stern R. 2014. Hyaluronan in wound healing: rediscovering a major player. Wound Repair Regen. 22: 579593.

5. Nguyen N, Kumar A, Chacko S, Ouellette RJ, Ghosh A. 2017. Human hyaluronic acid synthase-1 promotes malignant transformation via epithelial-to-mesenchymal transition, micronucleation and centrosome abnormalities. Cell Commun. Signal. 15(1): 48.

6. Stern R, Kogan G, Jedrzejas MJ, Soltes L. 2007. The many ways to cleave hyaluronan. Biotechnol. Adv. 25: 537-557.
7. Nishihara T, Morimoto Y. 2017. Evaluation of transfer media containing different concentrations of hyaluronan for human in vitro fertilization. Reprod. Med. Biol. 16: 349-353.

8. Marei WF, Ghafari F, Fouladi-Nashta AA. 2012. Role of hyaluronic acid in maturation and further early embryo development of bovine oocytes. Theriogenology 78: 670-677.

9. Stern R, Jedrzejas MJ. 2006. Hyaluronidases: their genomics, structures, and mechanisms of action. Chem. Rev. 106: 818839.

10. Kim E, Baba D, Kimura M, Yamashita M, Kashiwabara S, Baba T. 2005. Identification of a hyaluronidase, Hyal5, involved in penetration of mouse sperm through cumulus mass. Proc. Natl. Acad. Sci. U S A 102: 18028-18033.

11. Kimura M, Ishida K, Kashiwabara S, Baba T. 2009. Characterization of two cytoplasmic poly(A)-binding proteins, PABPC1 and PABPC2, in mouse spermatogenic cells. Biol. Reprod. 80: 545-554.

12. Yoon S, Chang KT, Cho H, Moon J, Kim JS, Min SH, et al. 2014. Characterization of pig sperm hyaluronidase and improvement of the digestibility of cumulus cell mass by recombinant pSPAM1 hyaluronidase in an in vitro fertilization assay. Anim. Reprod. Sci. 150: 107-114.

13. Myles DG, Primakoff P. 1984. Localized surface antigens of guinea pig sperm migrate to new regions prior to fertilization. J. Cell Biol. 99: 1634-1641.

14. Gmachl M, Kreil G. 1993. Bee venom hyaluronidase is homologous to a membrane protein of mammalian sperm. Proc. Natl. Acad. Sci. U S A 90: 3569-3573.

15. Park C, Kim YH, Lee SR, Park S, Jung Y, Lee Y, et al. 2018. Characterization of Recombinant Bovine Sperm Hyaluronidase and Identification of an Important Asn-XSer/Thr Motif for Its Activity. J. Microbiol. Biotechnol. 28: 1547-1553.

16. Geetha-Habib M, Park HR, Lennarz WJ. 1990. In vivo Nglycosylation and fate of Asn-X-Ser/Thr tripeptides. J. Biol. Chem. 265: 13655-13660.

17. Gabius HJ. 2018. The sugar code: Why glycans are so important. Biosystems 164: 102-111. 